\title{
Changing modality behaviour in the radio light curve of blazar PKS B1144 - 379
}

\author{
N. M. M. Said,${ }^{1,2 \star}$ S. P. Ellingsen ${ }^{\oplus},{ }^{1 \star}$ J. Liu ${ }^{\oplus},{ }^{3}$ J. N. McCallum, ${ }^{1}$ H. E. Bignall, ${ }^{2}$ S. Shabala ${ }^{\oplus \star}$ \\ and C. Reynolds ${ }^{2}$ \\ ${ }^{1}$ School of Natural Sciences, University of Tasmania, Private Bag 37, Hobart, TAS 7001, Australia \\ ${ }^{2}$ CSIRO Astronomy and Space Science, PO Box 1130, Bentley, WA 6102, Australia \\ ${ }^{3}$ Max-Planck-Institut für Radioastronomie, Auf dem Hügel 69, D-53121 Bonn, Germany
}

Accepted 2021 May 28. Received 2021 May 27; in original form 2020 October 12

\begin{abstract}
The highly variable BL Lac object PKS B1144 - 379 was monitored at $6.7 \mathrm{GHz}$ using the Ceduna Radio Telescope with high cadence from 2003 to 2011. Intraday variations due to interstellar scintillation (ISS) were observed throughout the period. To complement our earlier analysis of the ISS and variability of this object, we have investigated the physical origin of changes in the modality of flux density distributions, calculated for $\sim 14 \mathrm{~d}$ observing blocks. Our analysis shows that the flux density distribution is primarily bimodal, but it changes to unimodal during the core brightening and jet expansion phases of the source. The presence of unimodal flux density distributions during these two phases is most likely due to the compactness of the scintillating component and the intrinsic evolution of the source. The existence of unimodality in the flux density distributions associated with specific phases of the source evolution also suggests that changes in the modality are unlikely due to multiple scattering screens. We propose that the physical origin of changes in the modality of the flux density distribution for PKS B1144 - 379 is most likely due to the combination of multiple bright jet features with interstellar scintillation along the line of sight between observer and source. This new approach complements our previous investigations of the temporal evolution of PKS B1144 - 379 that used interstellar scintillation and very long baseline interferometry, and the combination of these techniques provides a crucial starting point for understanding the system.
\end{abstract}

Key words: methods: statistical-quasars: individual: PKS B1144-379.

\section{INTRODUCTION}

Statistical techniques have been widely used in the analysis of astronomical data sets to detect clustering in certain characteristic quantities. Studying the distribution of data can provide a crucial starting point for understanding the system as well as the underlying mechanism. Numerous examples exist in the literature, covering more than 25 yr. These include Zinn (1985), who found bimodality in the distribution of metallicities in a sample of 121 Milky Way globular clusters, and Ashman, Bird \& Zepf (1994), who discussed a number of statistical techniques and used the Kernel Density Estimator Mixture Model (KMM) algorithm to estimate the statistical significance of bimodality and clustering in globular cluster metallicity distributions, the velocity distributions of galaxies in clusters, and gamma-ray burst durations, while Nemec \& Nemec (1991, 1993) used mixture modelling to investigate the number of distinct stellar populations in the Milky Way.

Bimodality of a distribution has been found in a range of astrophysical contexts using a variety of analysis techniques. Bimodality in the distribution of gamma-ray burst durations was discovered by Kouveliotou et al. (1993). Liodakis et al. (2017) presented a

^E-mail: noor.mdsaid@utas.edu.au (NMMS); simon.ellingsen@utas.edu.au (SPE); stanislav.shabala@utas.edu.au (SS) maximum likelihood approach for detecting the bimodal trait by examining the $15 \mathrm{GHz}$ blazar radio flux variability observed using the Owens Valley Radio Observatory. The differences in variability characteristics between BL Lacs, FSRQs, and between gamma-ray detected and non-detected sources were also investigated. RojasArriagada et al. (2019) used APOGEE spectroscopic survey data to investigate the distribution of the $\mathrm{Mg}$ abundance (as a tracer of the alpha elements)-versus-metallicity plane of a sample of stars selected to be in the inner region of the bulge, showing that the distribution in the $[\mathrm{Mg} / \mathrm{Fe}]$-versus- $[\mathrm{Fe} / \mathrm{H}]$ plane is bimodal. Larrodera \& Cid (2020) used the bi-Gaussian function to separate the solar wind at $1 \mathrm{AU}$ into two main components, slow and fast. Yu et al. (2020) found evidence of two distinct types of short gamma-ray burst observed by Swift/Bat. A recent investigation by Vallely et al. (2020) used the presence of a bimodal velocity distribution in the ${ }^{56} \mathrm{Ni}$ decay products to infer that some Type Ia supernova ( $\mathrm{SNe}$ Ia) explosions are produced through white dwarf-white dwarf collisions.

We have previously used interstellar scintillation and very long baseline interferometry (VLBI) techniques in Paper I (Said et al. 2020) and Paper II (N.M.M. Said et al., submitted), respectively, to examine the extrinsic and intrinsic changes in PKS B1144 - 379. PKS B1144 - $379\left(11^{\mathrm{h}} 47^{\mathrm{m}} 01.4^{\mathrm{s}},-38^{\circ} 12^{\prime} 11^{\prime \prime} \mathrm{J} 2000\right)$ is classified as a BL-Lac object due to its variability in optical, infrared, and radio wavelengths (Nicolson et al. 1979; Stickel, Fried \& Kuehr 1989). 
Véron-Cetty \& Véron (2006) identified this source as a quasar. It has a redshift $z=1.048$ (Stickel et al. 1989) and was categorized as a radio variable in the Parkes $2700 \mathrm{MHz}$ survey by Bolton \& Shimmins (1973). It is known to exhibit both long-term and short-term flux density variations at centimetre wavelengths, which are caused by source intrinsic evolution and interstellar scintillation along the line of sight between the observer and the source (Kedziora-Chudczer et al. 2001a, b; Shabala, Santoso \& Godfrey 2012b). In this paper we present a statistical analysis of the centimetre continuum flux density distribution over a $\sim 14$-d period of PKS B1144 - 379 obtained from COSMIC [Continuous Single Dish Monitoring of intraday variability (IDV) at Ceduna] data observed at $6.7 \mathrm{GHz}$ between 2003 and 2011 (McCulloch et al. 2005; Blanchard 2013). The purpose of this further work is to investigate the physical origin of changes in the modality of flux density distribution and to establish whether there is a relationship with either intrinsic or extrinsic effects.

The structure of this paper is as follows. The COSMIC data are described in Section 2. In Section 3 and Section 4, we present the intrinsic evolution of PKS B1144 - 379 and our statistical analyses. In Section 5, we discuss the potential causes of a bimodal flux density distribution. Results and discussion are presented in Section 6 and Section 7. We summarize our conclusions in Section 8.

\section{DATA}

In Papers I and II we give a detailed description of the measurements of PKS B1144 - 379 taken from the COSMIC project (McCulloch et al. 2005; Blanchard 2013) between 2003 and 2011. We have used the same data in the current investigation and further details on the single dish data and its analysis can be found in Paper I. One 'block' of PKS B1144 - 379 flux density data typically corresponds to observations over a $\sim 14$-d period. We have flux density data for the calibrator and target source for most blocks at least once per hour when they were above 10 degrees elevation for the Ceduna antenna. Analysis of the data over periods of this duration make it possible to compare changes in the properties of the flux density variability over time. Over the $9 \mathrm{yr}$ of the COSMIC project a total of 101 independent blocks of flux density data were measured for PKS B1144 - 379. For the analysis presented in this paper we have used 67 of the 101 blocks of flux density data; excluded blocks consisted of data with gaps of three or more days.

\section{INTRINSIC EVOLUTION OF PKS B1144 - 379}

Fig. 1 shows the long-term variations in the mean flux density of PKS B1144 - 379 at $6.7 \mathrm{GHz}$ taken as part of the COSMIC observing project between 2003 and 2011. The flux density averaged over a block of data at this frequency was observed to vary between 0.8 to 3.4 Jy over the $9 \mathrm{yr}$ period. Shabala et al. (2012a) and Said et al. (2020) identified that two flares in the flux density of PKS B1144 - 379 occurred during this period, the first commenced in 2005 November (point C) and the second in 2008 August (point F). These two flares were identified in Paper I by examining the values of $\chi^{2}$ and the fitted parameters of the interstellar scintillation annual cycle. In Papers I and II, we reported that PKS B1144 - 379 flares approximately every 3-4 yr. The peak flux density in the $8.6 \mathrm{GHz}$ VLBI images from Radio Reference Frame Image Database (RRFID) ${ }^{1}$ and Astrogeo ${ }^{2}$ database observed between 1997 and 2018 as well as the COSMIC observing

\footnotetext{
${ }^{1}$ https://www.usno.navy.mil/USNO/astrometry/vlbi-products/rrfid

${ }^{2}$ http://astrogeo.org/vlbi-images/
}

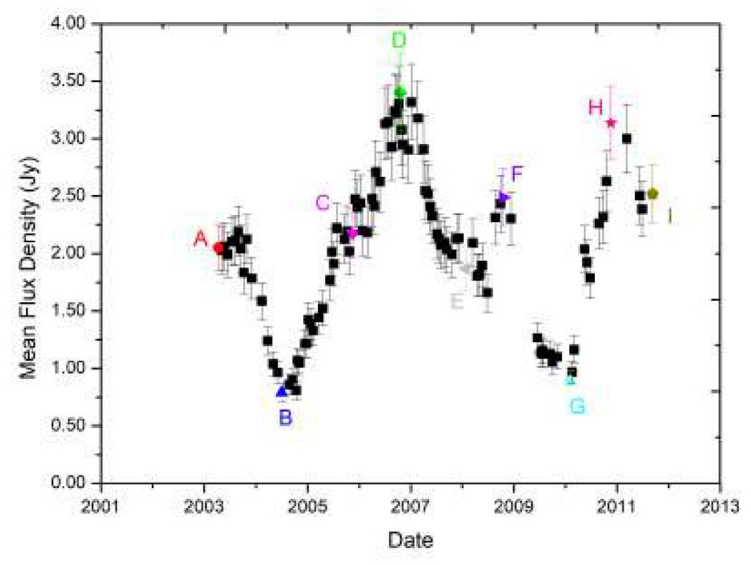

Figure 1. Long-term monitoring of the $6.7 \mathrm{GHz}$ flux density of PKS B1144 - 379 over the period 2003 through 2011. Here we used 101 independent blocks of mean flux density to show the source intrinsic variability. The total flux density of the source is divided into several phases; core brightening (segments B-C, E-F, and G-H), jet expansion (segment CD), and jet dispersion (segments A-B, D-E, F-G, and H-I). Two flares in total flux density were observed in 2005 November (point C) and 2008 August (point F), as reported in Paper I. Paper II identified two additional flares that occurred in 2002 September and 2010 November (point H). The error bars represent the error of measurement of the flux densities within each block. Data were taken from the COSMIC observing project.

project suggest that additional flares occurred in 2002 September and 2010 November, respectively. In Paper I we estimated the size of the scintillating component (presumably the AGN core, or a combination of the core and a compact part of the jet) of PKS B1144 - 379 using the 'Earth Orbital Synthesis' technique and found it ranged from 5.65 to $15.90 \mu$ as $(0.05-0.13 \mathrm{pc})$. Comparison with the modulation index of the flux density showed that the size of the scintillating component was at its most compact at the commencement of the two flares. On the basis of our investigations in Paper I and II we divided the long-term variations in the mean flux density of the source into several phases; core brightening (segments B-C, E-F, and G-H), jet expansion (segment C-D), and jet dispersion (segments A-B, D-E, F-G, and H-I). In general, the flaring event begins with the core increasing its brightness. After the flare, the shocked plasma knots will eventually extend past the core and begin to resolve during the jet expansion phase. The core flux density then decreases to its quiescent state throughout the jet dispersion phase as the shocked plasma knot propagates further down the jet. We refer the reader to Paper II for further details on the measurements and analysis of the source evolution phases.

\section{STATISTICAL ANALYSIS}

We have employed a number of statistical techniques to study changes in the flux density distribution of PKS B1144 - 379 and quantify the modality of the distribution.

\subsection{Distribution analysis}

Kernel density estimation (KDE) Multivariate ${ }^{3}$ is a non-parametric way to estimate the probability density function of a random variable.

${ }^{3}$ statsmodels.nonparametric.kernel-density.KDEMultivariate python package. 
It works by placing a kernel (a weighting function) that provides a measure of the density at each point in the data set and then summing the kernels to generate a kernel density estimate for the overall sample. Gaussian kernel density estimation (Rosenblatt 1956; Parzen 1962) was performed for each of the 67 blocks of flux density for PKS B1144 - 379 to identify clusters in the data. We used a cross-validation maximum likelihood method to select the kernel bandwidth (Habbema, Hermans \& Van Den Broek 1974; Duin 1976). The optimum bandwidth of $0.03 \mathrm{Jy}$ was selected by taking the mean value of the bandwidth estimated for all blocks and this was then used in our analysis.

We compared the Gaussian KDE for each block of flux density with the data from the block when PKS B1144 - 379 was in its quiescent (lowest flux density) state. The block observed at the most quiescent phase for PKS B1144-379 in 2004 July 2, point B in Fig. 1, was used as a reference block for comparison with all others. The Kolmogorov-Smirnov $(\mathrm{K}-\mathrm{S})^{4}$ statistic for a two-sample test was computed to determine whether the Gaussian KDE for each block differed significantly from that of the quiescent state. The $\mathrm{K}-\mathrm{S}$ test is a two-sided test for the null hypothesis that two independent samples are drawn from the same continuous distribution (Simard \& L'Ecuyer 2011). The $\mathrm{K}-\mathrm{S}$ test is non-parametric and has the advantage of making no assumptions about the underlying distribution of the data. If the $\mathrm{K}-\mathrm{S}$ statistic $D$ value is small or the p-value is high, then we cannot reject the hypothesis that the distributions of the two samples are the same.

\subsection{Determining the modality of a flux density distribution}

In the majority of cases, signatures of bimodality in a data set can seen by inspection of the distribution of a quantity. For example, if one can visually identify a double-peak in the Gaussian KDE of a flux density distribution, this suggests a bimodal profile. We utilized three methods to quantify potential modality in our flux density distributions: the Bimodality Coefficient (BC), Hartigan's Dip Statistic (HDS). and Pearson's $S$ - $K$ difference. These techniques were performed using the 'modes index', 'dip.test', and 'moments' packages in the $\mathrm{R}$ statistical analysis suite.

\subsubsection{Bimodality coefficient}

The $B C$ is a simple test to assess unimodality or multimodality in a data set (SAS Institute Inc 2004). The bimodality coefficient is easily calculated, requiring only the sample size, skewness, and excess kurtosis. A data set is considered to have a bimodal distribution if it has high skewness, low excess kurtosis, or both. The bimodality coefficient has a range of zero to one where a value greater than 0.555 suggests bimodality whereas lower values point toward unimodality. The $B C$ value is calculated as

$$
\mathrm{BC}=\frac{m_{3}^{2}+1}{m_{4}+3 \times\left((n-1)^{2} /(n-2)(n-3)\right)},
$$

where $n$ is the sample size, $m_{3}$ is the skewness of the distribution, and $m_{4}$ is the excess kurtosis estimated from the given data. Both the skewness and kurtosis are sensitive to sample bias therefore the corrected skewness and kurtosis for sample bias were used (Joanes \& Gill 1998; Doane \& Seward 2011; Pfister et al. 2013)

\footnotetext{
${ }^{4}$ scipy.stats.ks_2samp python package.
}

$$
\begin{aligned}
& m_{3}=\frac{\sqrt{n(n-1)}}{n-2}\left\{\frac{(1 / n) \sum_{i=1}^{n}\left(x_{i}-\bar{x}\right)^{3}}{\left(\sqrt{(1 / n) \sum_{i=1}^{n}\left(x_{i}-\bar{x}\right)^{2}}\right)^{3}}\right\} \\
& m_{4}=\frac{n-1}{(n-2)(n-3)} \cdot\left[(n+1)\left\{\frac{(1 / n) \sum_{i=1}^{n}\left(x_{i}-\bar{x}\right)^{4}}{\left((1 / n) \sum_{i=1}^{n}\left(x_{i}-\bar{x}\right)^{2}\right)^{2}}\right\}-3(n-1)\right],
\end{aligned}
$$

where $x_{i}$ and $\bar{x}$ represent the data and mean value.

\subsubsection{Hartigan's dip statistic}

The HDS test determines whether that data set contains more than one mode in the distribution using the dip statistic. The 'dip' refers to the maximum difference between the empirical distribution function and a unimodal distribution function that minimizes the maximum difference where a uniform distribution is used as a reference unimodal distribution (Hartigan \& Hartigan 1985). The mathematical definition and computational algorithm of dip are described in detail by Hartigan \& Hartigan (1985) and Hartigan (1985). The null hypothesis of the HDS test is that a distribution is unimodal. For the HDS measure, we used the p-value resulting from Hartigan's dip test. $H D S$ values range from 0 to 1 , with a p-value less than 0.05 indicating significant multimodality, a p-value greater than 0.05 but less than 0.10 suggesting multimodality with marginal significance, and p-value greater than 0.10 inferring unimodality (Freeman \& Dale 2013). Compared to the $B C$ test, the presence of positive skew biased $H D S$ values will result in a greater tendency overall for a distribution to be classified as multimodal.

\subsection{Pearson's $S$ - $K$ difference}

The Jarque-Bera test statistic (Jarque \& Bera 1987) can be used to analyse the whether or not a distribution is normal, categorizing the data as either normal or non-normal. This test is based on the relationship between skewness $(S)$ and kurtosis $(K)$.

$C_{1}=S^{2}+((K-3) / 2)^{2}$.

The quantity $C_{1}$ will be equal to zero for the normal distribution ( $S$ $=0, K=3$ ) and $9 / 25$ for a non-normal distribution. Therefore, we categorized the flux density distribution as normal for $C_{1} \leq 9 / 25$ whereas greater values point toward a non-normal distribution.

We then determined the modality of the flux density distribution using the Pearson's $S$ - $K$ difference (Pearson 1929).

$C_{2}=S^{2}-(K-3)$,

where we considered the flux density distributions as bimodal for values of $C_{2}$ greater than 1.2, marginally significant bimodality for values less than 1.2 but greater than 1.1, and being unimodal for $C_{2}$ less than 1.1 (Gaedke \& Klauschies 2017).

\section{POTENTIAL CAUSES OF A BIMODAL FLUX DENSITY DISTRIBUTION}

Potentially both extrinsic and intrinsic mechanisms can produce bimodality in the distribution of the flux density of PKS B1144 - 379. An example of an extrinsic mechanism would be interstellar scintillation where there are contributions from multiple scattering screens along the line of sight to the source. An example of an intrinsic mechanism is interstellar scintillation where there are contributions from multiple emission regions (e.g. the core and a compact bright 

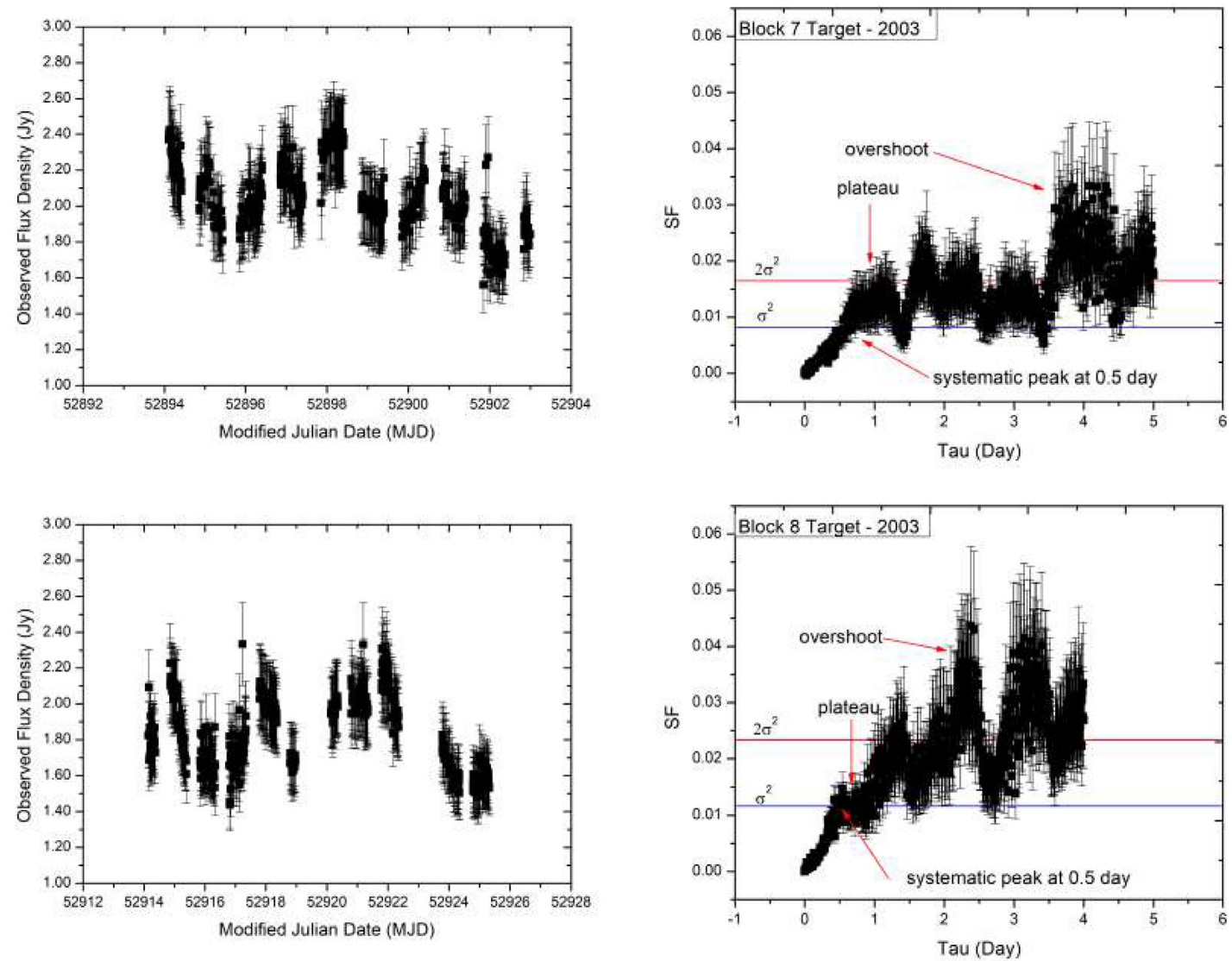

Figure 2. Light curves of PKS B1144 - 379 (left-hand panel) and its normalized structure functions (right-hand panel) observed on 2003 August 29 (top panel) and 2003 September 16 (bottom panel). The saturated value $\left(2 \sigma^{2}\right)$ and variance level are represented by the horizontal red and blue lines in the Structure Function (SF) of the target source. More information on the calculation and interpretation of the structure function can be found in Paper I. The modalities of these two flux density distributions, as measured by Pearson's $S$ - $K$ difference test, have changed from bimodal (on August 29) to unimodal (September 16).

jet feature). If there are multiple scattering screens along the line of sight to a source, we expect that to result in multiple variability timescales present in the structure function of the target source. Further details on the structure functions created from each 'block' of the flux density were discussed in Paper I. Fig. 2 shows an example of $\mathrm{a} \sim 12 \mathrm{~d}$ observing blocks of PKS B1144 - 379 (left-hand panel) and its normalized structure functions (right-hand panel) observed on 2003 August 29 (top panel) and 2003 September 16 (bottom panel). To investigate the possibility of multiple variability time-scales, we examined the structure function of each block for PKS B1144 - 379 for evidence of multiple time-scales that will result in multiple plateaus. Over the $9 \mathrm{yr}$ of COSMIC observations, we did not find any structure functions that clearly showed more than one plateau, suggesting that the interstellar scintillation for PKS B1144 - 379 is primarily due to a single scattering screen along the line of sight.

\section{RESULTS}

Fig. A1 shows the flux density distributions and the Gaussian KDEs of each block of PKS B1144 - 379 observed between 2003 and 2011. Only 67 out of 101 blocks of flux density were used in statistical analyses to improve the accuracy of the determination of modality. Data blocks with a gap of more than $3 \mathrm{~d}$ were excluded. Visual inspection of the Gaussian KDEs shows that the peak, shape, and modality of the flux density distributions changed over the observing period. These significant changes are supported by the results of the $\mathrm{K}-\mathrm{S}$ test where we are able to reject the null hypothesis that the distribution of the reference (quiescent phase) block is the same as that of the other blocks (p-value $\ll 0.01$; Simard \& L'Ecuyer 2011). The top panel of Fig. 3 shows the significant difference in the K-S test $D$ value between the Gaussian KDE of each block and the reference observed on 2004 July 2 (quiescent state at point B). The K-S statistical test $D$ value increases rapidly as the mean flux density increases from the quiescent level (point B) showing that the modality in the Gaussian KDE of the flux density distribution has become much more distinct (see Fig. 4). We examined whether the selection of the specific Gaussian KDE reference block impacts our results and found that the $D$ values for blocks in mid-2004 differ significantly from the rest of the data, regardless of the choice of the reference block.

We employed three different tests of modality for our data and here we compare the results from each to investigate the level of agreement between them. Fig. 5 shows the results obtained by performing the $B C$ test for each block of flux density. We designated each block as either showing bimodality (black points) or unimodality (red points) referring to bimodality coefficient values. This test finds that the majority of the flux density distributions exhibit bimodality with only 11 blocks of flux density classified as unimodal. Most of the unimodal flux density distributions are located within the core brightening and jet expansion phases (segments B-C, C-D, and E-F). Fig. 6 shows the classification of modality for each block of flux density using the $H D S$ test. The classification was determined by calculating the 


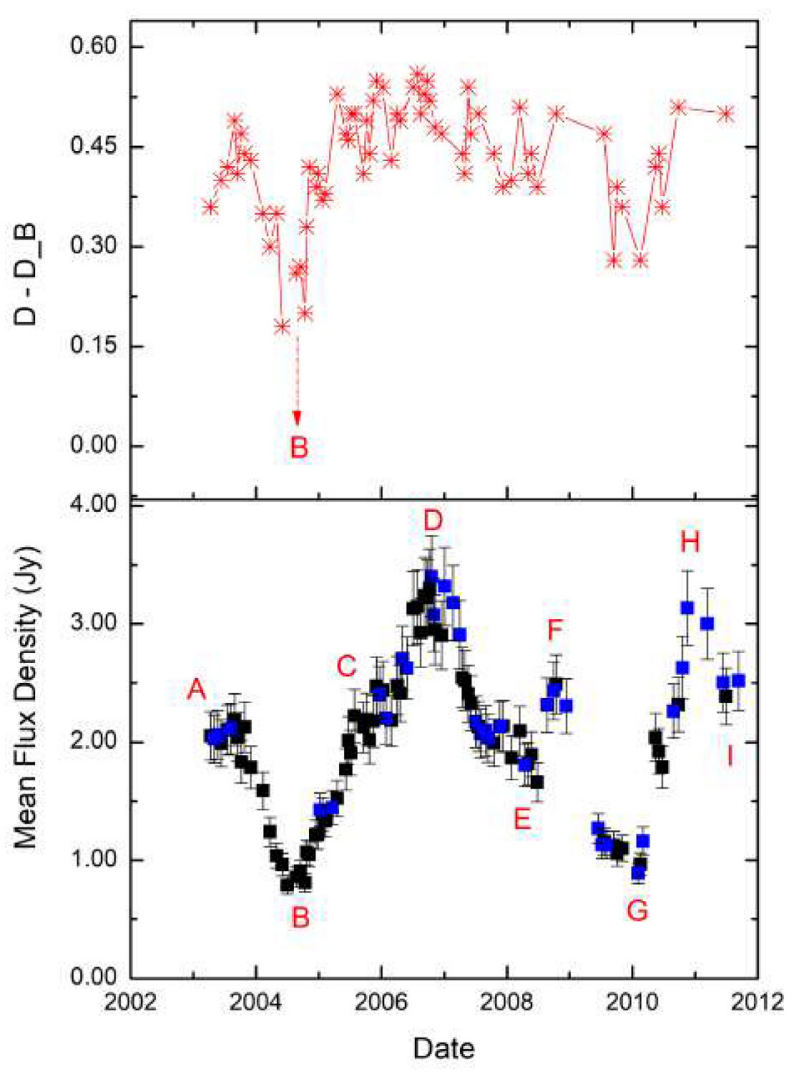

Figure 3. The top panel represents the $D$ statistic value for the $\mathrm{K}-\mathrm{S}$ test comparing the Gaussian KDE at the quiescent level (block of flux density observed on 2004 July 2 at point B) with each block of observations for PKS B1144 - 379. As indicated by the arrow, the $D$ statistic value of the quiescent level at point $B$ is equal to 0 . The bottom panel shows the long-term monitoring of the flux density for PKS B1144 - 379 over the period 2003 through 2011. Here we used 67 and 101 independent blocks of mean flux density for the $\mathrm{K}-\mathrm{S}$ test (top panel) and the long-term monitoring of the flux density (bottom panel), respectively. Blue points in the mean flux density plot represent the excluded blocks that consisted of data with gaps of three or more days.

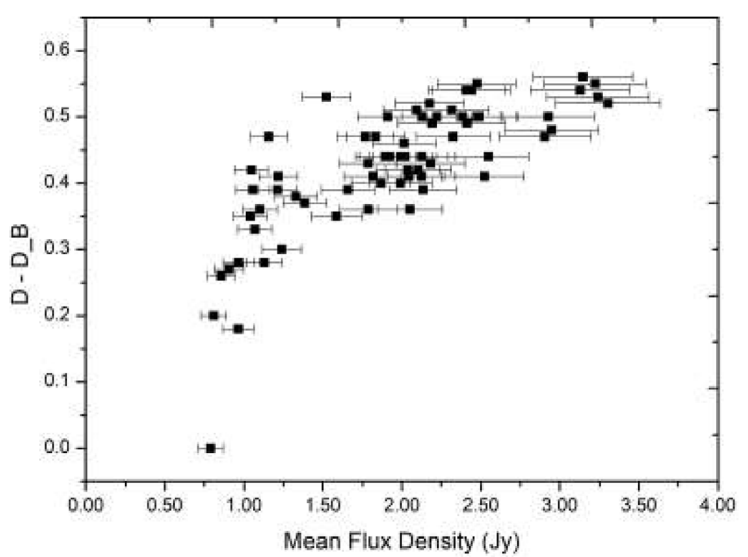

Figure 4. Correlation between the $D$ statistic values of PKS B1144 - 379 determined using the $\mathrm{K}-\mathrm{S}$ test versus its mean flux density. Here, we chose a block of flux density observed on 2004 July 2 at point B (quiescent level) as the reference distribution. The $D$ statistic value of the quiescent level at point $\mathrm{B}$ is equal to 0 . Only 67 independent blocks of mean flux density were used as we excluded data with a gap of more than $3 \mathrm{~d}$. Data were taken from the COSMIC observing project for the period from 2003 to 2011.

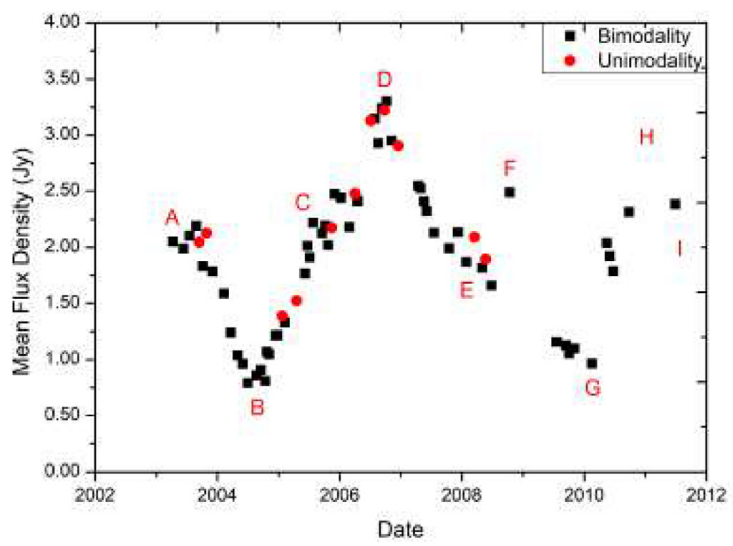

Figure 5. Determination of the modality for each block of flux density of PKS B1144 - 379 using the BC test. Black and red points represent bimodal and unimodal flux density distributions, respectively. Here, only 67 independent blocks of mean flux density were used as we excluded data with a gap of more than $3 \mathrm{~d}$. Data were taken from the COSMIC observing project for the period from 2003 to 2011 .

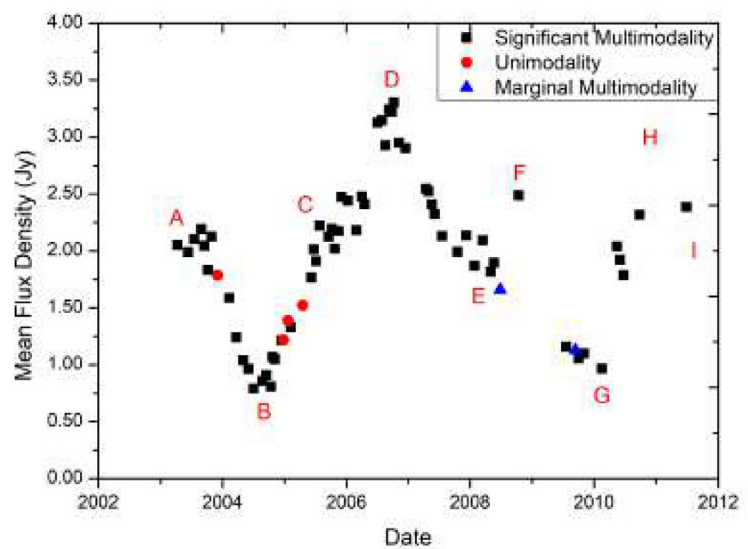

Figure 6. Determination of the modality for each block of flux density of PKS B1144 - 379 using the HDS test. Black, red, and blue points represent significant multimodal, unimodal, and marginal multimodal flux density distributions, respectively. Here, only 67 independent blocks of mean flux density were used as we excluded data with a gap of more than $3 \mathrm{~d}$. Data were taken from the COSMIC observing project for the period from 2003 to 2011.

p-value. Only four unimodal flux density distributions (red points) were identified, mostly during the core brightening phase (segment B-C) and two blocks of flux density show marginal multimodality (blue points). The rest of the blocks have flux density distributions with significant multimodal behaviour (black points). Determination of modality for each block of flux density using the Pearson's $S$ - $K$ difference test is shown in Fig. 7. For each block we have calculated the $C_{2}$ value (equation 5). We found 18 unimodal flux density distributions (red points) while the rest of blocks are bimodal (black points) with four marginal bimodal (blue points). Most of the unimodal flux density distributions were detected during the core brightening and jet expansion phase (segments B-C, C-D, E-F, and $\mathrm{G}-\mathrm{H})$. Table A1 gives the values for each of the tests for each of the 67 observing blocks. 


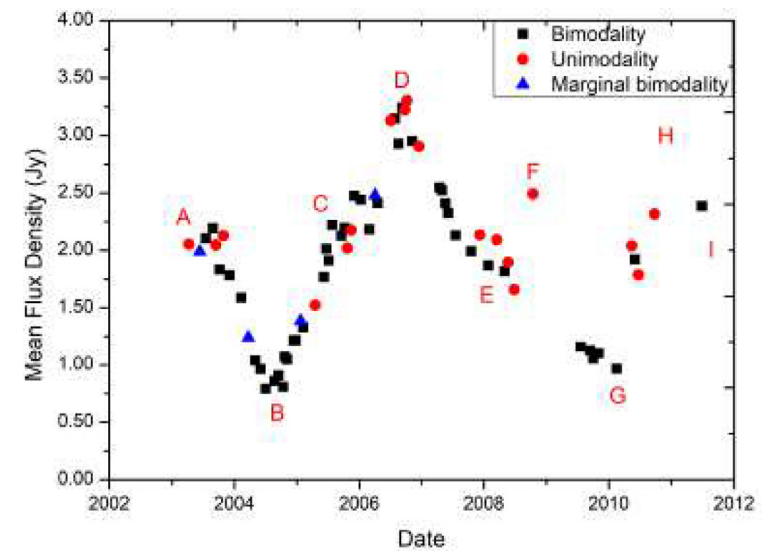

Figure 7. Determination of the modality for each block of flux density of PKS B1144 - 379 using the Pearson's $S$ - $K$ difference test. Black, red, and blue points represent bimodal, unimodal, and marginal bimodal flux density distributions, respectively. Here, only 67 independent blocks of mean flux density were used as we excluded data with a gap of more than $3 \mathrm{~d}$. Data were taken from the COSMIC observing project for the period from 2003 to 2011.

\section{DISCUSSION}

We have undertaken distribution analysis of the $6.7 \mathrm{GHz}$ flux density data for PKS B1144 - 379 observed between 2003 and 2011. The K-S test shows significant changes in the peak, shape, and modality of the flux density distributions over the observing period. Overall, the statistical analyses performed using the $B C, H D S$, and Pearson's $S$ - $K$ difference tests show similar patterns, although different classifications of modality have been identified for some blocks of flux density. These differences are thought to be due to the susceptibility of the $B C$ and $H D S$ tests to higher/positive skewness and excess kurtosis, as well as their assumptions/hypothesis of the underlying distributions. Determination of normality for the flux density distributions of PKS B1144 - 379 using the Jarque-Bera test statistic shows that 90 per cent of them are categorized as being a non-normal distribution. Therefore, we considered the Pearson's $S$ - $K$ difference, a non-parametric test, to be the most suitable test for determining the modality of our flux density distribution.

In Paper I, we found that the size of the scintillating component (presumably the core or a combination of the core and the compact part of the jet) of PKS B1144 - 379 was at its most compact at the beginning of the two flares (2005 November and 2008 August). The compactness of the scintillating component is associated with the modulation index of the flux density distribution, with a higher modulation index indicating a greater fraction of the source's emission is compact. During the jet expansion phase (segment C-D), the mean flux density increased due to the shocked plasma knots extending past the core and starting to resolve. In the meantime, the modulation index decreased and this implies that a lower fraction of the flux density arises from the most compact components (i.e. consistent with an expansion of the emission region). In this study, we find that unimodal flux density distributions were detected mostly during the core brightening and jet expansion phases (segments B-C, C-D, E-F, and G-H). The presence of unimodal flux density distributions during these two phases is likely associated with the compactness of the scintillating component and the source intrinsic evolution. These results complement our investigations using VLBI imaging and scintillation techniques (Papers I and II), which suggested flares in 2002 September and 2010 November (point H), respectively. The existence of unimodality in the flux density distributions associated with particular phases of the source evolution also suggests that changes in the modality are unlikely due to multiple scattering screens, assuming that multiple scattering screen positions are fixed along the source line of sight. The entanglement of the intrinsic (jet extended component) and extrinsic (interstellar scintillation) effects will significantly affect our ability to determine the cause of changes in the modality of flux density distribution. Therefore, we assume that the physical origin of changes in the modality of the flux density distribution for PKS B114 - 379 is most likely due to the combination of multiple bright jet features with interstellar scintillation occurring along the line of sight between the observer and source.

The existence of bimodality in most of the flux density distributions of PKS B1144 - 379 is in good agreement with the detection of bimodal behaviour in the $15 \mathrm{GHz}$ blazar radio variability observed using the Owens Valley Radio Observatory (Liodakis et al. 2017). These results also suggested that a model that takes into account the bimodality of the sources could possibly improve the study of the underlying flux density distribution. The results we have obtained for PKS B1144 - 379 suggests that distribution analysis of well-sampled flux density data may provide a new method to study of the evolution of the most compact radio sources. This new technique complements the 'Earth Orbital Synthesis' analysis of the scintillation time-scale and the VLBI imaging techniques we used in Paper I and II to investigate source evolution. Modeling flux density distribution and scintillation effect could also be done in the future to improve our understanding of the underlying process. Comparison between IDV and intrahour variability (IHV) radio sources with different timescales and fractional changes in flux density could be made to confirm that the changes in modality do correlate with intrinsic evolution in the same way as for PKS B1144 - 379. We predict that IHV sources, which are typically less extreme in terms of fractional changes in flux density than PKS B1144 - 379, will have a much higher fraction of unimodal flux density distributions dominated by emissions from a single compact component.

The potential to utilize single dish monitoring of sources to predict when a flare is likely to occur will facilitate optimal planning of observing strategies for astronomical VLBI imaging and also geodetic observations. Geodetic observations utilize extragalactic radio sources, quasars, as reference points when measuring Earth's shape and orientation in space. Quasars, however, are not pointlike sources and their internal structure will degrade the accuracy of the observations and ultimately, accuracy of the final product, the global reference frame (Schaap et al. 2013). It is therefore important to understand the evolution of the radio source in order to enhance the modelling of the source structure that we use in geodetic observations.

\section{CONCLUSIONS}

The highly variable BL Lac object PKS B1144 - 379 was monitored at $6.7 \mathrm{GHz}$ using the Ceduna Radio Telescope with high cadence from 2003 to 2011. Intraday variations due to interstellar scintillation (ISS) were observed throughout the period. To complement our earlier analysis of the ISS and variability of this object, we have investigated the physical origin of changes in the modality of flux density distributions, calculated for $\sim 14$-d observing blocks. Total of 67 blocks of Gaussian KDE for each block of PKS B1144 - 379 were compared with the data from the block when this source was at its quiescent (lowest flux density) state. The statistical analysis carried out using the $\mathrm{K}-\mathrm{S}$ test indicates that there are significant changes in its peak, shape, and modality. An evaluation of three 
different methods of assessing the modality within each block were conducted and the results show that $B C, H D S$, and Pearson's $S$ $K$ difference tests show similar patterns. The marginal difference in modality due to the susceptibility of the $B C$ and $H D S$ tests to higher/positive skewness and excess kurtosis, as well as their assumptions/hypothesis of the underlying distributions suggests that the Pearson $S$ - $K$ difference is the most reliable indicator of modality for data with our characteristics. We deduced that the flux density distribution is mainly bimodal, but it changes to unimodal during the core brightening and jet expansion phase of the source. The presence of unimodal flux density distributions during these two phases is most likely due to the compactness of the scintillating component and the intrinsic evolution of the source. The existence of unimodality in the flux density distributions associated with particular phases of the source evolution also suggests that changes in the modality are unlikely due to multiple scattering screens, assuming that multiple scattering screen positions are fixed along the source line of sight. We suggest that the physical origin of changes in the modality of the flux density distribution for PKS B1144 - 379 is most likely due to interplay between multiple bright jet features and interstellar scintillation occurring along the line of sight between the observer and source. Analysis of the flux density distribution appears to be a promising technique for the study of the dynamical evolution of the quasar and may provide a new means to detect the onset of a flare.

\section{ACKNOWLEDGEMENTS}

We would like to thank Prof Ue-Li Pen and Dr Artem V. Tuntsov for helpful discussions. We acknowledge L. Petrov who maintains the Astrogeo archive and all contributors to this dataset. The National Radio Astronomy Observatory is a facility of the National Science Foundation, operated under cooperative agreement by Associated Universities, Inc. This research has made use of the United States Naval Observatory (USNO) RRFID, and the NASA/ IPAC Extragalactic Database (NED), which is operated by the Jet Propulsion Laboratory, California Institute of Technology, under contract with the National Aeronautics and Space Administration. N.M.M.Said acknowledges the Astronomical Society of Australia (ASA) for providing travel grant that facilitated some of the work undertaken for this paper.

\section{DATA AVAILABILITY}

The Ceduna data sets generated during and/or analysed during the current study are available from the corresponding author, N.M.M.Said, upon request.

\section{REFERENCES}

Ashman K. M., Bird C. M., Zepf S. E., 1994, AJ, 108, 2348 Blanchard J. M., 2013, PhD thesis, University of Tasmania Bolton J. G., Shimmins A. J., 1973, Aust. J. Phys. Astrophys. Suppl., 30, 1 Doane D. P., Seward L. E., 2011, J. Stat. Educ., 19 n2 Duin R. P. W., 1976, IEEE Trans. Comput., 25, 1175

Freeman J. B., Dale R., 2013, Behav. Res. Methods, 45, 83 Gaedke U., Klauschies T., 2017, Limnol. Oceanogr.: Methods, 15, 979 Habbema J. D. F., Hermans J., Van Den Broek K., 1974, in Compstat 1974. Proceedings in Computational Statistics. Physica Verlag, Wien, p. 101 Hartigan J. A., Hartigan P. M., 1985, Ann. Statist., 13, 70 Hartigan P. M., 1985, J. R. Stat. Soc., 34, 320
Jarque C. M., Bera A. K., 1987, Int. Stat. Rev., 55, 163

Joanes D. N., Gill C. A., 1998, J. R. Stat. Soc., 47, 183

Kedziora-Chudczer L., Jauncey D. L., Wieringa M. A., Tzioumis A. K., Bignall H. E., 2001a, Ap\&SS, 278, 113

Kedziora-Chudczer L. L., Jauncey D. L., Wieringa M. H., Tzioumis A. K., Reynolds J. E., 2001b, MNRAS, 325, 1411

Kouveliotou C., Meegan C. A., Fishman G. J., Bhat N. P., Briggs M. S., Koshut T. M., Paciesas W. S., Pendleton G. N., 1993, ApJ, 413, L101

Larrodera C., Cid C., 2020, A\&A, 635, A44

Liodakis I., Pavlidou V., Hovatta T., Max-Moerbeck W., Pearson T. J., Richards J. L., Readhead A. C. S., 2017, MNRAS, 467, 4565

McCulloch P. M., Ellingsen S. P., Jauncey D. L., Carter S. J. B., Cimò G., Lovell J. E. J., Dodson R. G., 2005, AJ, 129, 2034

Nemec J. M., Nemec A. F. L., 1991, PASP, 103, 95

Nemec J. M., Nemec A. F. L., 1993, AJ, 105, 1455

Nicolson G. D., Glass I. S., Feast M. W., Andrews P. J., 1979, MNRAS, 189, 29P

Parzen E., 1962, Ann. Math. Statist., 33, 1065

Pearson K., 1929, Biometrika, 21, 370

Pfister R., Schwarz K. A., Janczyk M., Dale R., Freeman J. B., 2013, Front. Psychol., 4, 700

Rojas-Arriagada A., Zoccali M., Schultheis M., Recio-Blanco A., Zasowski G., Minniti D., Jönsson H., Cohen R. E., 2019, A\&A, 626, A16

Rosenblatt M., 1956, Ann. Math. Statist., 27, 832

Said N. M. M., Ellingsen S. P., Bignall H. E., Shabala S., McCallum J. N., Reynolds C., 2020, MNRAS, 498, 4615

Inc SAS Institute, 2004, SAS/ETS 9.1 User's Guide, 1-7. SAS Institute Inc., Cary, NC

Schaap R. G., Shabala S. S., Ellingsen S. P., Titov O. A., Lovell J. E. J., 2013, MNRAS, 434, 585

Shabala S., Titov O., Lovell J., McCallum J., Blanchard J., Watson C., Dickey J., 2012a, in Baver D.,Behrend D. K., eds, Seventh General Meeting (GM2012) of the international VLBI Service for Geodesy and Astrometry (IVS). National Aeronautics and Space Administration, Madrid, Spain, p. 329

Shabala S. S., Santoso J. S., Godfrey L. E. H., 2012b, ApJ, 756, 161

Simard R., L'Ecuyer P., 2011, J. Stat. Softw. Artic., 39, 1

Stickel M., Fried J. W., Kuehr H., 1989, A\&AS, 80, 103

Vallely P. J., Tucker M. A., Shappee B. J., Brown J. S., Stanek K. Z., Kochanek C. S., 2020, MNRAS, 492, 3553

Véron-Cetty M.-P., Véron P., 2006, A\&A, 455, 773

Yu Y. B., Li L. B., Li B., Geng J. J., Huang Y. F., 2020, New Astron., 75, 101306

Zinn R., 1985, ApJ, 293, 424

\section{SUPPORTING INFORMATION}

Supplementary data are available at MNRAS online.

Figure A1. Radio variability of PKS B1144 - 379 and the estimated probability density function.

Please note: Oxford University Press is not responsible for the content or functionality of any supporting materials supplied by the authors. Any queries (other than missing material) should be directed to the corresponding author for the article.

\section{APPENDIX: STATISTICAL RESULTS}



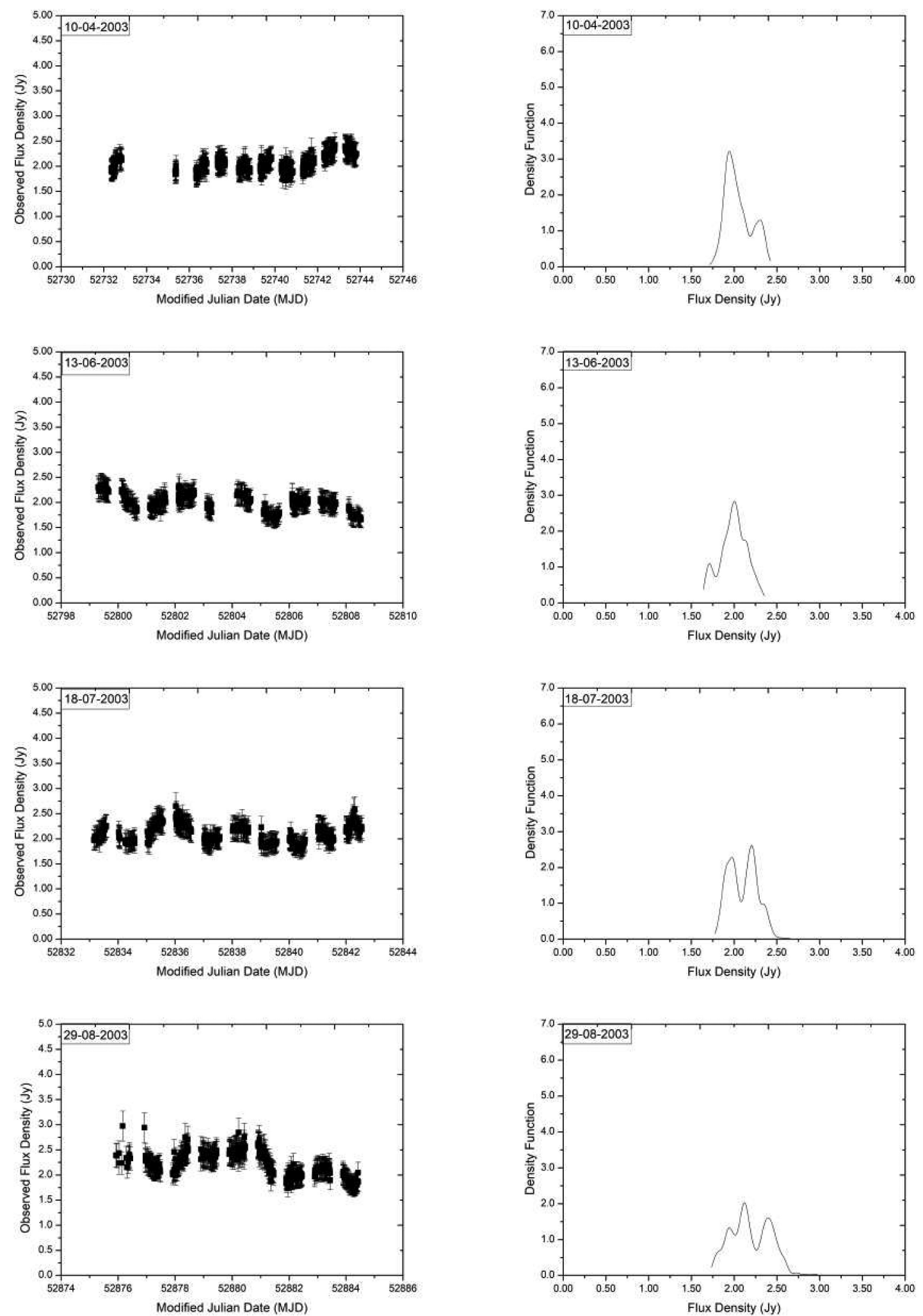

Figure A1. Radio variability of PKS B1144 - 379 and the estimated probability density function. The remaining panel for the period 2003-2011 are available online. Data taken from the COSMIC observing project observed between 2003 to 2011. 
Table A1. Statistical results of the Ceduna data set at $6.7 \mathrm{GHz}$ for PKS B1144 - 379. Columns from left to right: (1) observing date, (2) mean flux density, (3) uncertainty of mean flux density, (4) $K-S$ test, (5) Bimodality coefficient test (BC), (6) modality of flux density distribution measured using $B C$ where B and U represent the bimodality (p-value $>0.555$ ) and unimodality (p-value $<0.555)$, respectively, (7) Hartigan's dip statistic test (HDS), (8) modality of flux density distribution measured using HDS test where SM, MM, and U represent significant multimodality (p-value $<0.05)$, marginal multimodality $(0.05<\mathrm{p}$-value $<$ 0.10 ), and unimodality (p-value $>0.10)$, respectively), (9) skewness $(S),(10)$ kurtosis $(K),(11) C_{1},(12)$ normality of flux density distribution measured using Jarque-Bera test statistic where $N$ and NN represent normal $\left(C_{1} \leq 9 / 25\right)$ and non-normal $\left(C_{1}>9 / 25\right),(13) C_{2}$ and (14) modality measured using Pearsons's $S$ - $K$ difference where $\mathrm{B}, \mathrm{MB}$, and $\mathrm{U}$ represent bimodality $\left(C_{2}>1.2\right)$, marginal significant of bimodality $\left(1.2>C_{2}>1.1\right)$ and unimodality $\left(C_{2}<1.0\right)$, respectively.

\begin{tabular}{|c|c|c|c|c|c|c|c|c|c|c|c|c|c|}
\hline Date & $S(\mathrm{Jy})$ & $\sigma(\mathrm{Jy})$ & $\mathrm{K}-\mathrm{S}$ & $B C$ & Modality & $H D S$ & Modality & $S$ & $K$ & $C_{1}$ & Normality & $C_{2}$ & Modality \\
\hline 2003-06-13 & 1.99 & 0.16 & 0.40 & 0.575 & B & 0.03 & SM & 0.46 & 2.11 & 0.41 & $\mathrm{NN}$ & 1.1 & MB \\
\hline $2003-08-29$ & 2.19 & 0.23 & 0.49 & 0.553 & B & 0.05 & SM & 0.15 & 1.86 & 0.35 & $\mathrm{~N}$ & 1.2 & B \\
\hline 2003-09-16* & 2.04 & 0.19 & 0.41 & 0.533 & $\mathrm{U}$ & 0.04 & SM & 0.68 & 2.76 & 0.48 & $\mathrm{NN}$ & 0.7 & $\mathrm{U}$ \\
\hline 2003-10-07 & 1.83 & 0.20 & 0.47 & 0.575 & B & 0.05 & SM & -0.11 & 1.77 & 0.39 & NN & 1.2 & B \\
\hline $2003-10-29 *$ & 2.13 & 0.25 & 0.44 & 0.477 & $\mathrm{U}$ & 0.06 & SM & 0.74 & 3.24 & 0.56 & $\mathrm{NN}$ & 0.3 & $\mathrm{U}$ \\
\hline 2004-02-11 & 1.59 & 0.12 & 0.35 & 0.566 & B & 0.03 & SM & -0.17 & 1.82 & 0.37 & $\mathrm{NN}$ & 1.2 & B \\
\hline $2004-03-23$ & 1.24 & 0.11 & 0.30 & 0.826 & B & 0.03 & SM & 1.78 & 5.06 & 4.24 & $\mathrm{NN}$ & 1.1 & MB \\
\hline 2004-05-02 & 1.04 & 0.12 & 0.35 & 0.614 & B & 0.07 & SM & 0.04 & 1.64 & 0.47 & $\mathrm{NN}$ & 1.4 & B \\
\hline 2004-06-03 & 0.96 & 0.07 & 0.18 & 0.659 & B & 0.04 & SM & 0.26 & 1.63 & 0.54 & $\mathrm{NN}$ & 1.4 & B \\
\hline 2004-07-02 & 0.79 & 0.06 & 0.00 & 0.706 & B & 0.04 & SM & 0.38 & 1.63 & 0.61 & $\mathrm{NN}$ & 1.5 & B \\
\hline 2004-08-23 & 0.86 & 0.09 & 0.26 & 0.597 & B & 0.04 & SM & 0.15 & 1.72 & 0.43 & $\mathrm{NN}$ & 1.3 & B \\
\hline $2004-12-26 *$ & 1.22 & 0.12 & 0.41 & 0.738 & B & 0.02 & $\mathrm{U}$ & -0.63 & 1.91 & 0.69 & NN & 1.5 & B \\
\hline $2005-01-22^{*}$ & 1.39 & 0.13 & 0.37 & 0.541 & $\mathrm{U}$ & 0.02 & $\mathrm{U}$ & 0.15 & 1.90 & 0.32 & $\mathrm{~N}$ & 1.1 & MB \\
\hline 2005-02-07 & 1.33 & 0.12 & 0.38 & 0.715 & B & 0.08 & SM & -0.20 & 1.47 & 0.63 & $\mathrm{NN}$ & 1.6 & B \\
\hline 2005-04-19 & 1.52 & 0.24 & 0.53 & 0.492 & $\mathrm{U}$ & 0.01 & $\mathrm{U}$ & -0.55 & 2.66 & 0.33 & $\mathrm{~N}$ & 0.6 & $\mathrm{U}$ \\
\hline 2005-06-09 & 1.77 & 0.16 & 0.47 & 0.715 & B & 0.09 & SM & -0.01 & 1.41 & 0.63 & $\mathrm{NN}$ & 1.6 & B \\
\hline $2005-06-23$ & 2.01 & 0.18 & 0.46 & 0.709 & B & 0.07 & SM & 0.11 & 1.44 & 0.62 & $\mathrm{NN}$ & 1.6 & B \\
\hline 2005-07-07 & 1.91 & 0.25 & 0.50 & 0.743 & B & 0.04 & SM & 0.81 & 2.23 & 0.80 & $\mathrm{NN}$ & 1.4 & B \\
\hline 2005-07-27 & 2.22 & 0.24 & 0.50 & 0.753 & B & 0.04 & SM & 0.78 & 2.14 & 0.79 & $\mathrm{NN}$ & 1.5 & B \\
\hline 2005-09-19 & 2.13 & 0.17 & 0.41 & 0.617 & B & 0.06 & SM & 0.47 & 1.99 & 0.48 & $\mathrm{NN}$ & 1.2 & B \\
\hline 2005-10-07 & 2.19 & 0.21 & 0.49 & 0.663 & B & 0.05 & SM & 0.33 & 1.68 & 0.54 & $\mathrm{NN}$ & 1.4 & B \\
\hline $2005-10-24^{*}$ & 2.02 & 0.27 & 0.44 & 0.745 & B & 0.07 & SM & 1.70 & 5.24 & 4.16 & $\mathrm{NN}$ & 0.7 & $\mathrm{U}$ \\
\hline $2005-11-15^{*}$ & 2.18 & 0.36 & 0.52 & 0.473 & $\mathrm{U}$ & 0.04 & SM & 0.58 & 2.83 & 0.34 & $\mathrm{~N}$ & 0.5 & $\mathrm{U}$ \\
\hline 2006-09-10 & 3.24 & 0.28 & 0.53 & 0.607 & B & 0.06 & SM & 0.25 & 1.76 & 0.45 & $\mathrm{NN}$ & 1.3 & B \\
\hline $2006-09-25^{*}$ & 3.22 & 0.35 & 0.55 & 0.472 & $\mathrm{U}$ & 0.02 & SM & 0.64 & 2.99 & 0.41 & $\mathrm{NN}$ & 0.4 & $\mathrm{U}$ \\
\hline 2006-10-08* & 3.30 & 0.33 & 0.52 & 0.588 & B & 0.04 & SM & 0.81 & 2.83 & 0.67 & $\mathrm{NN}$ & 0.8 & $\mathrm{U}$ \\
\hline 2006-11-07 & 2.95 & 0.29 & 0.48 & 0.788 & B & 0.04 & SM & 1.07 & 2.74 & 1.16 & $\mathrm{NN}$ & 1.4 & B \\
\hline $2006-12-17^{*}$ & 2.90 & 0.25 & 0.47 & 0.503 & $\mathrm{U}$ & 0.06 & SM & 0.37 & 2.27 & 0.27 & $\mathrm{~N}$ & 0.9 & $\mathrm{U}$ \\
\hline 2007-04-16 & 2.55 & 0.19 & 0.44 & 0.740 & B & 0.03 & SM & 0.59 & 1.83 & 0.69 & $\mathrm{NN}$ & 1.5 & B \\
\hline 2007-04-30 & 2.52 & 0.16 & 0.41 & 0.575 & B & 0.04 & SM & 0.29 & 1.90 & 0.39 & $\mathrm{NN}$ & 1.2 & B \\
\hline $2007-05-21$ & 2.41 & 0.24 & 0.54 & 0.740 & B & 0.10 & SM & 0.00 & 1.36 & 0.67 & $\mathrm{NN}$ & 1.6 & B \\
\hline 2007-06-06 & 2.33 & 0.18 & 0.47 & 0.649 & B & 0.05 & SM & 0.16 & 1.59 & 0.52 & $\mathrm{NN}$ & 1.4 & B \\
\hline $2007-07-20$ & 2.13 & 0.20 & 0.50 & 0.696 & B & 0.07 & SM & -0.15 & 1.49 & 0.59 & $\mathrm{NN}$ & 1.5 & B \\
\hline $2007-10-18$ & 1.99 & 0.18 & 0.44 & 0.648 & B & 0.04 & SM & 0.46 & 1.88 & 0.52 & $\mathrm{NN}$ & 1.3 & B \\
\hline $2007-12-09^{*}$ & 2.14 & 0.20 & 0.39 & 0.574 & B & 0.03 & SM & 0.88 & 3.09 & 0.77 & NN & 0.7 & $\mathrm{U}$ \\
\hline 2008-01-27 & 1.87 & 0.14 & 0.40 & 0.643 & B & 0.05 & SM & 0.11 & 1.60 & 0.50 & $\mathrm{NN}$ & 1.4 & B \\
\hline $2008-03-16^{*}$ & 2.09 & 0.25 & 0.51 & 0.510 & $\mathrm{U}$ & 0.05 & SM & 0.07 & 1.99 & 0.26 & $\mathrm{~N}$ & 1.0 & $\mathrm{U}$ \\
\hline 2008-05-02 & 1.82 & 0.16 & 0.41 & 0.668 & B & 0.04 & SM & 0.58 & 2.02 & 0.58 & $\mathrm{NN}$ & 1.3 & B \\
\hline $2008-05-21^{*}$ & 1.90 & 0.22 & 0.44 & 0.522 & $\mathrm{U}$ & 0.05 & SM & 0.64 & 2.70 & 0.43 & NN & 0.7 & $\mathrm{U}$ \\
\hline $2008-06-26 *$ & 1.66 & 0.17 & 0.39 & 0.605 & B & 0.02 & MM & 0.73 & 2.54 & 0.59 & $\mathrm{NN}$ & 1.0 & $\mathrm{U}$ \\
\hline $2008-10-14^{*}$ & 2.49 & 0.29 & 0.50 & 0.561 & B & 0.03 & SM & 0.80 & 2.93 & 0.64 & NN & 0.7 & $\mathrm{U}$ \\
\hline 2009-07-21 & 1.16 & 0.17 & 0.47 & 0.644 & B & 0.04 & SM & -0.17 & 1.61 & 0.51 & $\mathrm{NN}$ & 1.4 & B \\
\hline
\end{tabular}


Table A1 - continued

\begin{tabular}{|c|c|c|c|c|c|c|c|c|c|c|c|c|c|}
\hline Date & $S$ (Jy) & $\sigma(\mathrm{Jy})$ & $\mathrm{K}-\mathrm{S}$ & $B C$ & Modality & $H D S$ & Modality & $S$ & $K$ & $C_{1}$ & Normality & $C_{2}$ & Modality \\
\hline 2009-09-13 & 1.13 & 0.11 & 0.28 & 0.725 & B & 0.02 & MM & 0.75 & 2.18 & 0.74 & $\mathrm{NN}$ & 1.4 & B \\
\hline 2009-10-02 & 1.06 & 0.14 & 0.39 & 0.644 & B & 0.03 & SM & 0.53 & 2.00 & 0.53 & $\mathrm{NN}$ & 1.3 & B \\
\hline 2009-11-03 & 1.10 & 0.15 & 0.36 & 0.743 & B & 0.03 & SM & 0.66 & 1.95 & 0.71 & $\mathrm{NN}$ & 1.5 & B \\
\hline $2010-02-16$ & 0.97 & 0.11 & 0.28 & 0.751 & B & 0.04 & SM & 0.51 & 1.70 & 0.68 & $\mathrm{NN}$ & 1.6 & $\mathrm{~B}$ \\
\hline $2010-05-16^{*}$ & 2.04 & 0.23 & 0.42 & 0.585 & B & 0.04 & SM & 1.02 & 3.48 & 1.09 & $\mathrm{NN}$ & 0.6 & $\mathrm{U}$ \\
\hline 2010-06-03 & 1.92 & 0.16 & 0.44 & 0.805 & B & 0.03 & SM & 1.03 & 2.57 & 1.10 & $\mathrm{NN}$ & 1.5 & B \\
\hline 2010-06-24* & 1.79 & 0.17 & 0.36 & 0.690 & B & 0.04 & SM & 1.36 & 4.16 & 2.20 & $\mathrm{NN}$ & 0.7 & $\mathrm{U}$ \\
\hline 2010-09-26* & 2.32 & 0.34 & 0.51 & 0.577 & B & 0.03 & SM & 0.78 & 2.79 & 0.62 & $\mathrm{NN}$ & 0.8 & $\mathrm{U}$ \\
\hline 2011-06-29 & 2.39 & 0.24 & 0.50 & 0.713 & B & 0.08 & SM & 0.26 & 1.52 & 0.62 & $\mathrm{NN}$ & 1.6 & B \\
\hline
\end{tabular}

Note. ${ }^{*}$ Distribution of flux density showing a different type of modality when comparing the results from all statistical analyses.

This paper has been typeset from a TEX/LATEX file prepared by the author. 